\title{
Improving transparency in observational social science research: A pre-analysis plan approach
}

\author{
Fiona Burlig*
}

October 30, 2017

\begin{abstract}
Social science research has undergone a credibility revolution, but these gains are at risk due to problematic research practices. Existing research on transparency has centered around randomized controlled trials, which constitute only a small fraction of research in economics. In this paper, I discuss three scenarios in which study preregistration can be credibly applied in non-experimental settings: cases where researchers collect their own data; prospective studies; and research using restricted-access data. Finally, I outline suggested contents for observational pre-analysis plans, and highlight where these plans should deviate from those designed for experimental research.
\end{abstract}

Keywords: Transparency, pre-registration, observational research JEL codes: A11, B41, C13

*Department of Economics and Energy Policy Institute, University of Chicago. Saieh Hall for Economics, 5757 S. University Avenue, Chicago, IL 60637. Email: burlig@uchicago.edu. I thank Patrick Baylis, Joshua Blonz, Garret Christensen, Hilary Hoynes, Jeremy Magruder, Edward Miguel, David Rapson, Reed Walker, and seminar participants at the Berkeley Initiative for Transparency in the Social Sciences for useful feedback. All remaining errors are my own. This research was generously supported by the National Science Foundation's Graduate Research Fellowship Program under grant DGE 1106400. Replication files for this project can be found at the Open Science Foundation: http://osf .io/4ft3w 


\section{Introduction}

In the wake of serious criticism of applied research (Hendry (1980), Sims (1980), Leamer (1983)), economics has undergone a credibility revolution (Angrist and Pischke (2010)). New data and an emphasis on research designs for causal inference has improved the rigor and believability of modern empirical research in economics. At the same time, there is a growing concern that existing research practices stand to undermine these gains (Ioannidis (2005), Miguel et al. (2014), Christensen and Miguel (forthcoming)). Several studies from across the social sciences have documented a variety of issues in existing research, including publication bias (Sterling (1959), Rosenthal (1979), Franco, Malhotra, and Simonovits (2014)); specification search (Simmons, Nelson, and Simonsohn (2011), Humphreys, de la Sierra, and van der Windt (2013), Brodeur et al. (2016); bias and failures to replicate (Open Science Collaboration (2015), Chang and Li (2015), Camerer et al. (2016), Ioannidis, Stanley, and Doucouliagos (2017)); and outright fraud (Simonsohn (2013) and Broockman, Kalla, and Aronow (2015)) 1 This research raises concerns about the validity of empirical work in economics and in social science more generally.

In light of this evidence, new research advancing transparency practices in the social sciences has begun to emerge (Miguel et al. (2014), Nosek et al. (2015), Christensen (2016), Christensen and Miguel (forthcoming)). One of the most popular tools for reducing publication bias and specification search in economics is study pre-registration using pre-analysis plans, wherein researchers submit a document describing the analysis they plan to carry out to a registry prior to accessing the data they will use. By creating a public record of proposed hypotheses, pre-analysis plans

1. See Christensen and Miguel (forthcoming) for a broad overview of problematic research practices in multiple social science fields, including economics. 
guard against the so-called "file drawer problem," and reduce ex post researcher degrees of freedom (Casey, Glennerster, and Miguel (2012), Finkelstein et al. (2012)). Study pre-registration is increasingly common in economics - since its inception in 2013, the American Economic Association's randomized controlled trial (RCT) registry has grown to include 1453 studies across 115 countries..$^{2}$ The nature of the registry itself is representative of the current state of pre-registration in economics: it only accepts pre-analysis plans for randomized controlled trials (RCTs). Up to this point, the vast majority of pre-registered research in economics has been for experimental designs. In general, registration-based methods for improving transparency in the social sciences have been targeted at experimental research alone, in large part because it is difficult to credibly pre-register observational work (Miguel et al. (2014), Christensen and Miguel (forthcoming)).

The challenges with pre-registering observational research are particularly problematic because only a small fraction of published papers in economics are experiments: in 2010, only $3 \%$ of papers in top journals were field RCTs (Card, DellaVigna, and Malmendier (2011)). Figure 1 presents the fraction of non-experimenta empirical papers published in the American Economic Review, the Journal of Political Economy, and The Quarterly Journal of Economics between 2005 and 2011, using data from Brodeur et al. (2016). Over this time period, non-experimental work across journals and fields - made up approximately 80 percent of published empirical research in these journals. As a result, addressing the credibility issues in empirical economics must involve advances for observational research.3

2. The registry can be found online at https://www.socialscienceregistry.org/; these numbers are current as of October 26, 2017.

3. Furthermore, researchers have suggested that pre-registration of experiments is not extremely valuable (Coffman and Niederle (2015), Olken (2015)), in part because there are strong norms around the presentation of results from papers using randomized controlled trials. The GoBifo ex- 
[Figure 1 about here]

Thus far, the only known example of a published observational economics paper that was credibly pre-registered is Neumark (2001), who prospectively specified an analysis to estimate the impacts of a federal minimum wage increase in the United States (Neumark (1999)). 4 $^{4}$ There is an active debate in the sciences about preregistration of observational work (see Epidemiology (2010), BMJ (2010), The Lancet (2010), PLOS Medicine (2014), Dal-Ré et al. (2014)). However, Christensen and Miguel (forthcoming) note that "there is often no credible way to verify that preregistration took place before analysis was completed...in our view, proponents of the pre-registration of observational work have not formulated a convincing response to this obvious concern."

In this paper, I contribute to the growing literature on research transparency in the social sciences by outlining three scenarios in which observational research can be credibly pre-registered. Researchers can use pre-analysis plans for observational research when they collect their own data - as in RCTs; when the study design involves events that have not yet occurred or the future public release of datasets; and when research involves restricted-access data. In these cases where researchers can credibly register analysis plans prior to accessing, pre-analysis plans can improve transparency in observational research. Credible pre-specification of observational data represents an important advance in transparency practices for social science.

periment detailed in Casey, Glennerster, and Miguel (2012) is an important empirical counterpoint to this argument.

4. See Christensen and Miguel (forthcoming) and Levine (2001) for more details on this example. Campolieti, Gunderson, and Riddell (2006) is a later paper in the same journal which uses the same empirical specification that was pre-specified by Neumark - though these researchers were unable to truly pre-specify their analysis, because their data were publicly available at the time the research was conducted. 
The remainder of this paper is organized as follows: Section 2 provides an overview of pre-analysis plans. Section 3 details the three situations in which researchers can credibly pre-register observational studies. Section 4 highlights areas where observational and experimental pre-analysis plans should differ. Section 5 provides a discussion of remaining challenges. Section 6 concludes.

\section{What is a pre-analysis plan?}

In general, a pre-analysis plan is a document wherein a researcher outlines her planned empirical analysis before having an opportunity to access her data. In order for the pre-analysis plan to be credible - uncontaminated by exploratory data analysis - the researcher needs to submit the pre-analysis plan to a (public) registry, which archives and timestamps the pre-analysis plan, preventing it from being altered after the researcher gains access to her data $5^{5}$ Pre-analysis plans are valuable tools for research transparency in two main ways. First, they contain a record of planned analyses, which allows consumers of research to observe hypotheses that are tested but do not appear in journal articles, guarding against publication bias. Second, pre-analysis plans "tie a researcher's hands" against (intentional or unintentional) specification search, data mining, "p-hacking," or selective presentation of hypotheses ${ }^{6}$ Finally, pre-analysis plans allow for credible adjustments for multiple

5. Prominent registries in the social sciences include the American Economic Association's RCT registry: http://www.socialscienceregistry.org, the Experiments in Governance and Politics registry: http://egap.org/design-registration/, the Registry for International Development Impact Evaluations: http://ridie.3ieimpact.org/, and the Open Science Framework: https: //osf.io/. Typically, these registries keep the contents of the pre-analysis plan private for some time after submission in order to protect researcher intellectual property.

6. Pre-analysis plans are not the only way to guard against these behaviors. Anderson and Magruder (2017) and Fafchamps and Labonne (2017) propose splitting the analysis sample into a "training" dataset and a "test" dataset. Researchers can perform exploratory analysis on the training data (which may include specification searching), but pre-register a set of analyses before 
hypothesis testing by laying the set of hypotheses that will be tested ex ante 7 While pre-analysis plans remain relatively uncommon in the social sciences, study preregistration has a successful history in the medical sciences, where registration of clinical drug trials is both mandatory for publication in top medical journals and required under U.S. law 8

\subsection{Current norms}

There are no established rules in the social sciences for exactly what a pre-analysis plan must contain. That said, there exist several useful overviews of suggested preanalysis plan contents, all written with RCTs in mind. Olken (2015) recommends including a primary outcome variable (or variables, with discussion of corrections for multiple inference); clear variable definitions that describe the creation of the variables from the raw data; rules for how the researcher will decide to exclude certain observations and handle missing data; empirical specifications (including covariates); heterogeneity analyses; and other issues of data monitoring. Glennerster and Takavarasha (2013) recommend describing the outcome measures (including making the distinction between primary and secondary outcomes); families that will be used for mean effects analysis; a description of subgroup analysis; the expected direction for one-sided statistical tests; and the main empirical specification. McKenzie (2012) recommends including a discussion of the sample; a description of the data sources;

accessing the test data. This approach has the desirable properties of allowing researcher "learning" and potentially improving power relative to a pre-analysis plan, but requires the test data to be credibly hidden from the researcher prior to the final analysis, which poses additional logistical challenges.

7. See Anderson (2008), Fink, McConnell, and Vollmer (2014), List, Shaikh, and Xu (2016), and Casey, Glennerster, and Miguel (2012) for further detail on multiple testing corrections.

8. The FDA's guidelines for medical study registration can be found at https://prsinfo. clinicaltrials.gov/definitions.html. 
the hypotheses to be tested; a description of variable construction; the estimating equation; plans for dealing with multiple hypothesis testing; plans for coping with survey attrition; addressing variables with limited variation; and the model to be tested. In general, economists tend to agree that pre-analysis plans must describe the outcomes of interest; the estimating equation or empirical approach; proposed heterogeneity analyses; and (where applicable) multiple testing corrections. In what follows, I discuss pre-analysis plans in the context of observational research.

\section{Credible pre-registration for observational research}

In order for a pre-analysis plan to improve transparency, researchers must be able to credibly demonstrate that it was registered prior to accessing the data. This has been a major barrier to pre-registration of observational research, because much observational research is performed on data that are already publicly available. There are, however, three key scenarios in which observational research can be credibly pre-specified: cases in which researchers collect their own data; prospective research studying future events or designed around future releases of public data; and research using restricted-access data. In all three cases, there are substantial barriers to accessing the data, which researchers can leverage to register a credible pre-analysis plan. In this section, I discuss each of these cases in further detail.

\subsection{Researcher-generated data}

A non-trivial amount of observational research involves researchers generating their own survey data. In these cases, as in RCTs, researchers can simply pre-register their analysis plan before data collection occurs. This is perhaps the easiest scenario 
in which to pre-register observational research, as researchers are intimately familiar with the structure of the dataset - and may even have conducted some pilot surveys - before getting access to the final version. When researchers collect their own data, worries about being "locked in" to an analysis plan only to find that a key variable is defined differently than expected ought to be assuaged. Despite the obvious overlap between cases where researchers collect their own data in RCTs and those where researchers collect their own data for quasi-experimental analysis, to the best of my knowledge, pre-specification of this type of observational research has not been discussed in the existing literature.

\subsection{Prospective analysis}

The one pre-analysis plan accompanying published observational research in economics is Neumark $(1999,2001)$. In this case, Neumark pre-registered an analysis plan for studying the impact of the October 1996 U.S. federal minimum wage increase. This pre-analysis plan included estimating equations, variable definitions, and groups for heterogeneity analysis, and was submitted to Industrial Relations prior to the May 1997 release of the Current Population Survey data that he planned to use. Neumark was therefore able to credibly register an observational research design by prospectively submitting his analysis plan before the release of the necessary data.

In Neumark's case, the pre-analysis plan was submitted before the data were released. In a recent example from political science, the Election Research Preacceptance Competition encouraged researchers to pre-register observational research on the 2016 general election prior to the March 2017 release of the ANES dataset..$^{9}$

9. More details can be found here: https://www.erpc2016.com//. 
Many datasets lend themselves to prospective pre-specification: both the American Census Bureau and IPUMS publish data release dates well in advance; and other research organizations such as the World Bank often announce when particular surveys are in the field. Better yet, many of the survey instruments used to generate these and other large administrative datasets are published in advance of the data being made available, which makes detailed pre-specification possible.

In addition to pre-specifying observational research prior to data being made publicly available, researchers can also credibly pre-specifiy observational research about future events. Researchers often know about policy changes or other economically meaningful events before they actually occur, and could therefore pre-specify an observational research design prior to the event.10 By pre-specifying a research design before the necessary data are released, or prior to a shock itself, researchers can credibly claim that they could not have explored the relevant data prior to registering the pre-analysis plan.

\subsection{Restricted access data}

Finally, research in economics is increasingly reliant upon proprietary, confidential, or restricted-access data. In the American Economic Review, for example, the fraction of papers requesting exemptions from the journal's open data policy rose from 6.5 percent in 2005 to over $45 \%$ by 2016 (the most recent year of data). Figure 2 displays the fraction of papers with exemptions over time. While this poses some challenges to transparency in general, it also represents an opportunity for pre-registered observational research. In general, researchers request access to these restricted data, which generates a time-stamped "paper trail." So long as the researcher registers

10. This would allow a researcher to pre-specify a design that leverages data that are made available in real time, such as information on social media platforms. 
her pre-analysis plan prior to being granted access to a confidential or otherwise restricted dataset, she can credibly pre-specify her empirical approach. Sharing confidential data usually comes with severe penalties, so in most cases, it is unlikely for researchers to be able to engage with the data prior to being granted official access.

[Figure 2 about here]

Furthermore, researchers are usually required to submit a document detailing what they wish to do with the data in order to gain access. In many cases, expanding this into a formal pre-analysis plan will involve relatively little work. It is easy to imagine employing this method when using confidential census microdata (e.g. Currie et al. (2015), Isen, Rossin-Slater, and Walker (2017)); when using data from a healthcare provider, utility, or other corporation (e.g. Mas and Moretti (2009), Handel (2013), and Ito (2014)); or any other type of data controlled by a nondisclosure agreement.

In some cases, it may also be possible to credibly pre-specify observational research using data that are available for purchase. In principle, any time where there is a significant barrier to accessing the necessary data, researchers can prespecify observational research, but the burden remains on the researcher to prove that she has not had access to the data prior to writing her pre-analysis plan. ${ }^{11}$ In the case of data that are for sale, researchers should pre-specify their analysis prior to buying the data, and should append the pre-analysis plan with a copy of the data purchase receipt, dated after the original plan was filed. The more expensive the data are, and the more severe are the penalties for sharing the data, the more credible this approach becomes.

11. Simply having to fill out an online form prior to downloading data, as is required to access various World Bank datasets, would likely not constitute a sufficient barrier to entry, because the costs of data access are so low., because these data are easy to access. 


\section{Observational pre-analysis plans}

In most respects, pre-analysis plans for observational research will look very similar to their experimental counterparts. There are, however, several key areas where observational plans should likely differ, especially when the observational design uses data that are not collected by the researcher herself. In this section, I discuss key features of pre-analysis plans, and describe areas where observational pre-analysis plans should deviate from the more traditional versions used for experimental research.

\subsection{Hypotheses}

Every pre-analysis plan should include a detailed outline of the hypotheses of interest. It can be helpful to further distinguish between main and alternative hypotheses in a pre-analysis plan (Casey, Glennerster, and Miguel (2012)). Ideally, pre-analysis plans should detail the model being tested, and describe the pattern of results that would allow the researcher to distinguish between different models. While this is a high bar, a carefully thought-out and pre-specified theoretical framework is very powerful. In a similar vein, it can also be beneficial to pre-specify tests for mechanisms if possible. A description of how these tests relates to the main hypotheses is useful. Pre-specifying mechanisms is not specific to observational research. In general, prespecified hypotheses need not look any different in observational research than in pre-analysis plans for RCTs.

\subsection{Data}

In both experimental and observational pre-analysis plans, researchers should include a detailed description of the data, including a discussion of how the outcome and 
treatment variables are constructed, and furthermore, which outcome variables will be used to test each hypothesis. This can prove challenging when researchers do not collect their own data, but is critical for motivating the chosen research design and reducing researcher degrees of freedom. Wherever possible, an observational pre-analysis plan should include a discussion of additional variables that will be used in the analysis. This section should also describe the circumstances that enable the researcher to register her pre-analysis plan prior to accessing the data.

\subsection{Research designs and empirics}

Without the benefit of randomization, observational studies rely on research designs to provide credible estimates of the causal effect of a given "treatment" on outcomes of interest. Researchers using quasi-experimental approaches often have many degrees of freedom in these designs, including which design to use, how exactly to implement the design, and which alternative specifications and robustness checks they will implement. As a result, carefully detailing the research design in the pre-analysis plan is particularly important for observational studies, as researchers conducting RCTs tend to have fewer degrees of freedom. ${ }^{12}$ Ideally, the research design section of an observational pre-analysis plan closely resembles the analogous section of the resulting paper, and include sufficient detail to guard against specification search or selective presentation of results. In addition, observational pre-analysis plans should discuss planned heterogeneity analyses, outline robustness checks, and describe statistical inference.

12. See, for instance, Olken (2015) for a discussion of this point. 


\subsubsection{Primary research design}

In particular, an observational pre-analysis plan should contain a detailed description of the chosen research design, including the identifying assumptions required for internal validity. Researchers should ensure that they pre-specify the important decisions that are involved in attempting to satisfying these assumptions. Observational pre-analysis plans should include a detailed description of the estimating equation that the researcher will use, and should indicate which specification (or set of specifications) is preferred. If a researcher plans to use different methods or samples to test different hypotheses, researchers should be clear about this. Preanalysis plans should clearly indicate which, if any, control variables will be used in the main analysis. In a regression discontinuity (RD) design, for example, the researcher should describe the running variable, appropriate threshold, functional form assumptions, bandwidth (or the use of an optimal bandwidth algorithm) and any additional controls required for identification. Researchers should also clearly describe the estimation sample.

\subsubsection{Validity checks}

Alongside the research design itself, researchers should also pre-specify tests that they will use to ensure the credibility of the research design. This may include placebo and falsification exercises, as well as additional research-design-specific checks. Continuing the $\mathrm{RD}$ example from above, the researcher could specify a series of covariate smoothness tests and a McCrary (2008) density test. Ideally, the researcher will also include a description of changes to the analysis that would be required if one of these tests were to fail. If, for instance, there are two RD thresholds, but there is evidence of severe manipulation in the first stage for one threshold, the researcher 
could decide to only use the second threshold - and pre-specify this decision. In experimental research, a similar example would be pre-specifying a check for covariate balance, and controlling for any variable in which the difference between treatment and control groups is statistically significant at the 95 percent level. In some cases, it may also be useful to pre-specify robustness checks, such as including key explanatory variables as controls, where researchers have substantial freedom to decide between ways of demonstrating robustness ex post. The list of robustness checks in a pre-analysis plan should not be intended to be exhaustive; referees and others will always ask researchers to perform additional checks, but laying out a framework for robustness checks of interest ex ante can help researchers demonstrate that they are not selectively presenting the results of these tests.

\subsubsection{Heterogeneity}

Beyond the main research design, pre-analysis plans should include a thorough description of heterogeneity analysis, in part because sub-group splits are often particularly vulnerable to specification search. A heterogeneity section in a pre-analysis plan this might include, for example, (but need not be limited to) subsetting the sample population by baseline covariates and re-running the analysis, including interaction terms in regression analysis, or performing a test using only the later years of the data. When possible, pre-specifying a model in conjunction with heterogeneity analysis can be particularly powerful, as differential effects are often useful to distinguish between models. This type of pre-specified additional analysis could easily find a place in pre-analysis plans for experimental research as well, but may be especially useful in observational work where researchers typically have access to larger samples than in randomized experiments, and may be well-powered to detect heterogeneous 
impacts $\sqrt{13}$ Pre-specification of heterogeneity analysis is also useful in cases where the intervention of interest was expected to have differential impacts in different subsets of the population.

\subsection{Inference}

Statistical inference is a key part of pre-analysis plans, both for observational and experimental designs. Each specified hypothesis test should have an accompanying inference procedure. This has the potential to increase the statistical power available to a researcher: using a pre-analysis plan allows for the credible use of one-sided statistical tests, for instance. Beyond the type of test used, researchers should also pre-specify how they will be calculating their standard errors. If they will be using Huber-White robust standard errors, Conley HAC standard errors, or other error adjustments such as clustering, this should be made clear in the pre-analysis plan. A pre-analysis plan that specifies using a two-way clustered standard error estimator, for example, should be sure to specify the dimensions of clustering.

Pre-analysis plans also allow for credible multiple-inference corrections, because readers have access to the full set of tests that a researcher has performed (or at least, intended to perform ex ante). In addition, common methods for adjusting for multiple testing, including Family-Wise Error Rate correction and grouping outcomes into indices, require researchers to define "families" of outcome variables that should be considered together. Pre-specification allows researchers to credibly generate this grouping prior to seeing how these choices impact statistical significance levels, thereby reducing degrees of freedom in this type of correction. Both Casey,

13. This need not be the case (see, for instance, Muralidharan and Niehaus (2017) for an overview), but is often true. 
Glennerster, and Miguel (2012) and Finkelstein et al. (2012) discuss the use of these multiple inference methods in pre-analysis plans.

\subsection{Conditionality}

Pre-analysis plans improve the transparency and credibility of social science research by forcing scholars to commit to a set of analyses before seeing the data. This does not, however, preclude pre-analysis plans from including conditional statements, which can increase the flexibility of a pre-analysis plan and prevent researchers from being forced to conduct analysis with obvious problems. Conditionality can be built into a pre-analysis plan throughout any of the sections described above, and may be particularly helpful in the case of observational studies where researchers are not collecting their own data.

Olken (2015) and McKenzie (2012) suggest one type of conditionality that can be pre-specified: the decision about what to do with problematic observations. How to clean data is an important decision, and if two researchers starting with the same raw dataset make different cleaning choices, they can end up with different outcomes, and researchers can build in conditional statements ("If any observation fails to report income, we will exclude it from the analysis") in order to aid data cleaning. Importantly, these data cleaning choices can be relative to the observed data (ie, dropping all outliers above the 99th percentile of income).

This can also take the form of a "data-adaptive" approach, wherein the analysis

is updated according to what is found in the data in a pre-specified manner. These approaches allow researchers to pre-specify decision rules, rather than exact choices, which enables additional flexibility without compromising transparency. The example above, of controlling for a covariate with a baseline imbalance, is a simple type 
of data-adaptive approach. In a more complex example, the Sustainable East Africa Research in Community Health RCT aimed at reducing the prevalence of HIV/AIDS in Africa used a pre-specified machine learning algorithm to determine which control variables to include in the final analysis in order to increase statistical precision (Balzer et al. (2015)). This is an RCT-based example, but similar approaches could be used in observational research. A cheeky reader might point out that taken to the extreme, the conditionality argument would suggest a decision rule of "report the results that are statistically significant," thus rendering the pre-analysis plan moot. In fact, this can be a legitimate (and transparent) decision rule, provided that the researcher also pre-specifies the correct adjustment for multiple inference and carries these out in her tests for statistical significance.

Conditionality becomes especially important in the case of observational work where a researcher is not collecting her own data. Suppose that a researcher can do an aggregate analysis with certainty, but to do a further disaggregated analysis requires combining two datasets, which may prove difficult. In this case, a researcher could pre-specify a decision rule detailing how the match will be performed, and how high the resulting match quality would have to be in order to go forward with the disaggregated analysis. Building in this type of conditionality affords the researcher some pre-specified flexibility. Importantly, this means that if a given condition on a dataset fails, the researcher can continue to do credible and transparent research - the alternative would be choosing ex post whether to continue with the analysis based on match quality, which re-introduces researcher degrees of freedom. There will of course always be issues that researchers cannot anticipate, but careful thought about the types of challenges that are likely to arise with a given observational dataset can help protect credibility if issues do arise. 


\section{Discussion}

It is important to recognize that not every observational study can be credibly prespecified. Pre-registration should not, therefore, be broadly required for observational research. There are many examples of excellent observational research that leverages publicly available existing data where a pre-analysis plan would have provided little-to-no benefit. The goal of encouraging more pre-registration in observational research is to improve transparency in situations where this is feasible, rather than to cast dispersions on work where this is not possible.

Similarly, like Christensen and Miguel (forthcoming), I strongly believe that ex post deviations from pre-analysis plans should be permitted. From a practical standpoint, pre-analysis plans can include mistakes or omit estimation that is clearly in keeping with the remainder of the analysis in the plan. When this happens, researchers should simply present all of the pre-specified research, and should also include corrected or forgotten analyses as well. In addition, researchers stand to learn a great deal from exploratory work. Having a pre-analysis plan for a paper's main hypotheses should in no way preclude researchers from conducting additional analysis - or from including this additional work in a paper alongside pre-specified research. In these cases, researchers simply need to clearly indicate which results were not pre-specified 14

14. Finkelstein et al. (2011), for example, do this by assigning a special character $\left({ }^{\wedge}\right)$ to non-prespecified results, and mention this at the beginning of the text, allowing readers to search (visually

or electronically) for these symbols. In the final version of the text (Finkelstein et al. (2012)), all of the non-pre-registered tests can be found in the appendix. 


\section{Conclusion}

Ensuring that the credibility revolution in empirical economics is not undermined by questionable research practices requires new work on research transparency methods for the social sciences. The majority of existing research in this area has focused on randomized controlled trials which, while an important component of modern economics research for their casual identification benefits, still represent only a small fraction of empirical research in economics. Improving transparency in economics more broadly will require new approaches to transparency that can be used in observational research.

In this paper, I suggest three settings in which pre-analysis plans, tools which guard against publication bias and limit researcher degrees of freedom, can credibly be used in observational research: cases where researchers collect their own data; prospective studies; and research using restricted-access data. The use of restrictedaccess data in particular is rapidly gaining in popularity, so pairing transparency methods with these types of data has the potential to generate significant progress towards improved transparency in non-experimental empirical research in economics. Future research should seek to develop new techniques - such as split-sample methods - that can be used to perform exploratory analysis on part of a dataset prior to preregistration, and to further develop "data-adaptive" approaches to pre-specification, allowing pre-analysis plans to be both detailed and flexible enough to accommodate researcher learning. 


\section{References}

Anderson, Michael L. 2008. "Multiple Inference and Gender Differences in the Effects of Early Intervention: A Reevaluation of the Abecedarian, Perry Preschool, and Early Training Projects." Journal of the American Statistical Association 103 (484): 1481-1495.

Anderson, Michael L., and Jeremy Magruder. 2017. "Split-Sample Strategies for Avoiding False Discoveries." National Bureau of Economic Research Working Paper No. 23544.

Angrist, Joshua D., and Jörn-Steffen Pischke. 2010. "The Credibility Revolution in Empirical Economics: How Better Research Design Is Taking the Con out of Econometrics." Journal of Economic Perspectives 24 (2): 3-30.

Balzer, Laura B., Maya L. Petersen, Mark J. van der Laan, and SEARCH Consortium. 2015. "Adaptive pair-matching in randomized trials with unbiased and efficient effect estimation." Statistics in Medicine 34 (6): 999-1011.

BMJ. 2010. "Registration of observational studies." BMJ 340:950.

Brodeur, Abel, Mathias Lé, Marc Sangnier, and Yanos Zylberberg. 2016. "Star Wars: The Empirics Strike Back." American Economic Journal: Applied Economics 8 (1): $1-32$.

Broockman, David, Joshua Kalla, and Peter Aronow. 2015. Irregularities in LaCour (2014). Working Paper.

Camerer, Colin F., Anna Dreber, Eskil Forsell, Teck-Hua Ho, Jürgen Huber, Magnus Johannesson, Michael Kirchler, et al. 2016. "Evaluating replicability of laboratory experiments in economics." Science 351 (6280): 1433-1436.

Campolieti, Michele, Morley Gunderson, and Chris Riddell. 2006. "Minimum Wage Impacts from a Prespecified Research Design: Canada 1981-1997." Industrial Relations: A Journal of Economy and Society 45 (2): 195-216.

Card, David, Stefano DellaVigna, and Ulrike Malmendier. 2011. "The Role of Theory in Field Experiments." Journal of Economic Perspectives 25 (3): 39-62.

Casey, Katherine, Rachel Glennerster, and Edward Miguel. 2012. "Reshaping Institutions: Evidence on Aid Impacts Using a Preanalysis Plan." The Quarterly Journal of Economics 127 (4): 1755-1812. 
Chang, Andrew C., and Phillip Li. 2015. "Is Economics Research Replicable? Sixty Published Papers from Thirteen Journals Say 'Usually Not'." FEDS Working Paper No. 2015-083.

Christensen, Garret. 2016. "Manual of Best Practices in Transparent Social Science Research." Working Paper.

Christensen, Garret S., and Edward Miguel. Forthcoming. "Transparency, Reproducibility, and the Credibility of Economics Research." Journal of Economic Literature.

Coffman, Lucas C., and Muriel Niederle. 2015. "Pre-analysis Plans Have Limited Upside, Especially Where Replications Are Feasible." Journal of Economic Perspectives 29 (3): 81-98.

Currie, Janet, Lucas Davis, Michael Greenstone, and Reed Walker. 2015. "Environmental Health Risks and Housing Values: Evidence from 1,600 Toxic Plant Openings and Closings." American Economic Review 105 (2): 678-709.

Dal-Ré, Rafael, John P. Ioannidis, Michael B. Bracken, Patricia A. Buffler, An-Wen Chan, Eduardo L. Franco, Carlo La Vecchia, and Elisabete Weiderpass. 2014. "Making Prospective Registration of Observational Research a Reality." Science Translational Medicine 6 (224): 224cm1.

Epidemiology. 2010. "The Registration of Observational Studies - When Metaphors Go Bad." Epidemiology 21 (5): 607-609.

Fafchamps, Marcel, and Julien Labonne. 2017. "Using Split Samples to Improve Inference on Causal Effects." Political Analysis: 1-18.

Fink, Gunther, Margaret McConnell, and Sebastian Vollmer. 2014. "Testing for heterogeneous treatment effects in experimental data: false discovery risk and correction procedures." Journal of Development Effectiveness 6 (1): 44-57.

Finkelstein, Amy, Sarah Taubman, Bill Wright, Mira Bernstein, Jonathan Gruber, Joseph P. Newhouse, Heidi Allen, Katherine Baicker, and Oregon Health Study Group. 2012. "The Oregon Health Insurance Experiment: Evidence from the First Year." The Quarterly Journal of Economics 127 (3): 1057-1106. 
Finkelstein, Amy, Sarah Taubman, Bill Wright, Mira Bernstein, Jonathan Gruber, Joseph P. Newhouse, Heidi Allen, Katherine Baicker, and The Oregon Health Study Group. 2011. "The Oregon Health Insurance Experiment: Evidence from the First Year." National Bureau of Economic Research Working Paper No. 17190 .

Franco, Annie, Neil Malhotra, and Gabor Simonovits. 2014. "Publication bias in the social sciences: Unlocking the file drawer." Science 345 (6203): 1502-1505.

Glennerster, Rachel, and Kudzai Takavarasha. 2013. Running Randomzied Evaluations: A Practical Guide. Princeton University Press.

Handel, Benjamin R. 2013. "Adverse Selection and Switching Costs in Health Insurance Markets: When Nudging Hurts." American Economic Review 103 (7): 2643-2682.

Hendry, David F. 1980. "Econometrics - Alchemy or Science?" Economica 47 (188): $387-406$.

Humphreys, Macartan, Raul Sanchez de la Sierra, and Peter van der Windt. 2013. "Fishing, Commitment, and Communication: A Proposal for Comprehensive Nonbinding Research Registration." Political Analysis 21 (1): 1-20.

Ioannidis, John P. A. 2005. "Why Most Published Research Findings Are False." PLoS Med 2 (8): e124.

Ioannidis, John P. A., T.D. Stanley, and Hristos Doucouliagos. 2017. "The Power of Bias in Economics Research." Economic Journal 127 (605): F236-F265.

Isen, Adam, Maya Rossin-Slater, and W. Reed Walker. 2017. "Every Breath You Take - Every Dollar You'll Make: The Long-Term Consequences of the Clean Air Act of 1970." Journal of Political Economy 125 (3): 848-902.

Ito, Koichiro. 2014. "Do Consumers Respond to Marginal or Average Price? Evidence from Nonlinear Electricity Pricing." American Economic Review 104 (2): 53763.

Leamer, Edward E. 1983. "Let's Take the Con Out of Econometrics." American Economic Review 73 (1): 31-43.

Levine, David I. 2001. "Editor's Introduction to "The Unemployment Effects of Minimum Wages: Evidence from a Prespecified Research Design"." Industrial Relations: A Journal of Economy and Society 40 (2): 161-162. 
List, John A., Azeem M. Shaikh, and Yang Xu. 2016. "Multiple Hypothesis Testing in Experimental Economics." National Bureau of Economic Research Working Paper No. 21875.

Mas, Alexandre, and Enrico Moretti. 2009. "Peers at Work." American Economic Review 99 (1): 112-45.

McCrary, Justin. 2008. "Manipulation of the running variable in the regression discontinuity design: A density test." Journal of Econometrics 142 (2): 698-714.

McKenzie, David. 2012. "A pre-analysis plan checklist." Impact Evaluations. Accessed August 25, 2015. http://blogs.worldbank.org/impactevaluations/ a-pre-analysis-plan-checklist.

Miguel, E., C. Camerer, K. Casey, J. Cohen, K. M. Esterling, A. Gerber, R. Glennerster, et al. 2014. "Promoting Transparency in Social Science Research." Science 343 (6166): 30-31.

Muralidharan, Karthik, and Paul Niehaus. 2017. "Experimentation at Scale." National Bureau of Economic Research Working Paper No. 23957.

Neumark, David. 1999. "The Employment Effects of Recent Minimum Wage Increases: Evidence from a Pre-specified Research Design." National Bureau of Economic Research Working Paper No. 7171.

. 2001. "The Employment Effects of Minimum Wages: Evidence from a Prespecified Research Design." Industrial Relations: A Journal of Economy and Society 40 (1): 121-144.

Nosek, B. A., G. Alter, G. C. Banks, D. Borsboom, S. D. Bowman, S. J. Breckler, S. Buck, et al. 2015. "Promoting an open research culture." Science 348 (6242): 1422-1425.

Olken, Benjamin A. 2015. "Promises and Perils of Pre-analysis Plans." Journal of Economic Perspectives 29 (3): 61-80.

Open Science Collaboration. 2015. "Estimating the reproducibility of psychological science." Science 349 (6251): aa4716.

PLOS Medicine. 2014. "Observational Studies: Getting Clear about Transparency." PLOS Medicine 11 (8): e1001711.

Rosenthal, Robert. 1979. "The file drawer problem and tolerance for null results." Psychological Bulletin 86 (3): 638-641. 
Simmons, Joseph P., Leif D. Nelson, and Uri Simonsohn. 2011. "False-Positive Psychology Undisclosed Flexibility in Data Collection and Analysis Allows Presenting Anything as Significant." Psychological Science 22 (11): 1359-1366.

Simonsohn, Uri. 2013. "Just Post It The Lesson From Two Cases of Fabricated Data Detected by Statistics Alone." Psychological Science 10 (24): 1875-1888.

Sims, Christopher A. 1980. "Macroeconomics and Reality." Econometrica 49 (1): 148.

Sterling, Theodore D. 1959. "Publication Decisions and Their Possible Effects on Inferences Drawn from Tests of Significance - or Vice Versa." Journal of the American Statistical Association 54 (285): 30-34.

The Lancet. 2010. "Should protocols for observational research be registered?" The Lancet 375 (9712): 348. 
Figure 1: Non-experimental papers represent the bulk of economics research
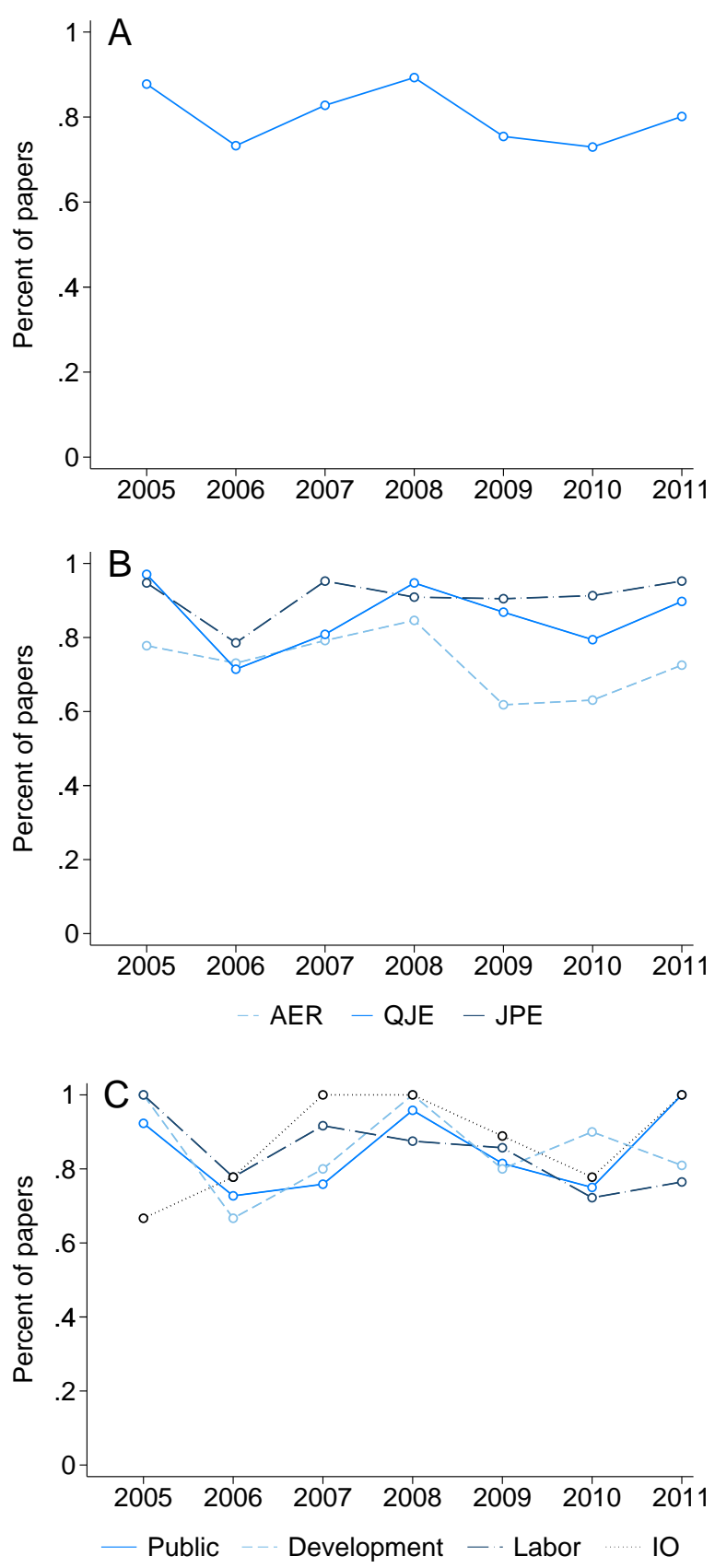

Notes: This figure presents the fraction of empirical papers that appeared in the American Economic Review, The Quarterly Journal of Economics, and the Journal of Political Economy that used nonexperimental research designs (ie, were not lab experiments or RCTs). The data are taken from Brodeur et al. (2016). Panel A presents data for the full sample; Panel B shows the data by journal, and panel $\mathrm{C}$ shows the data across four large microeconomics fields. In all cases, the majority of papers are non-experimental. 
Figure 2: Data policy exemptions are increasing over time

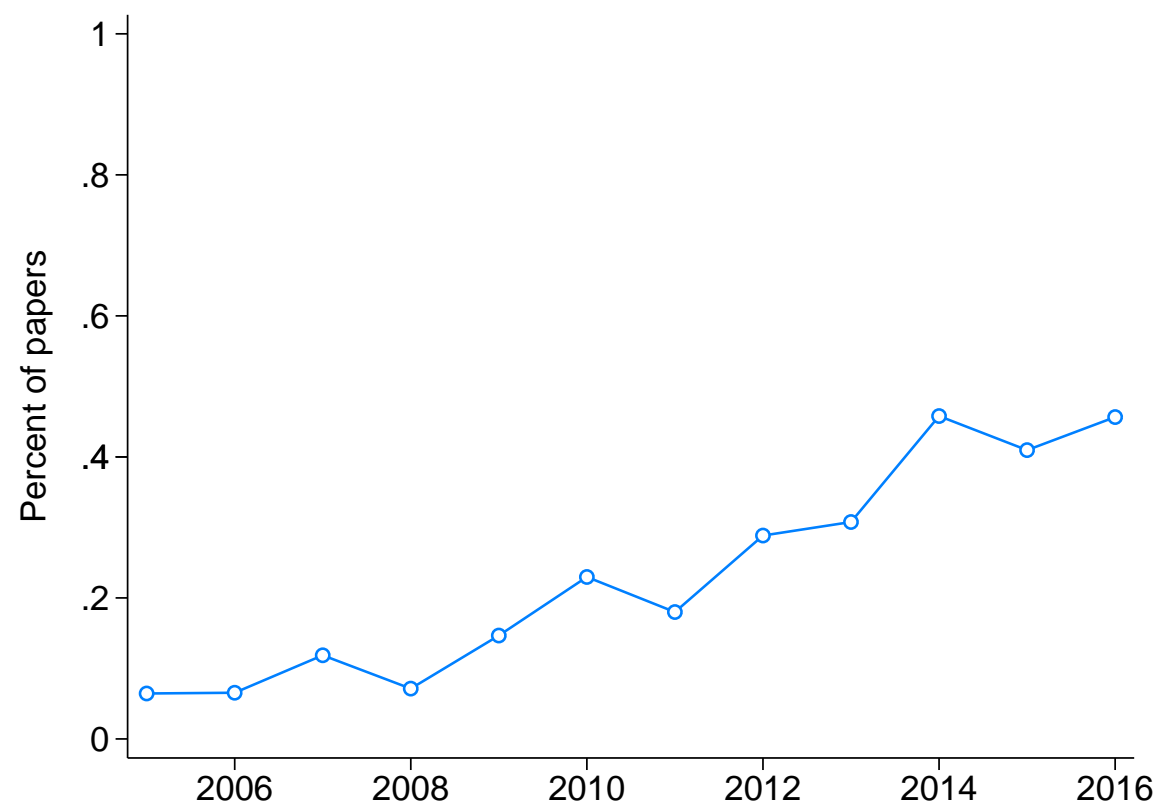

Notes: This figure presents the fraction of papers in the American Economic Review that had an exemption from the journal's data policy as a share of all empirical papers in the journal. I collected the data from the May (Papers and Proceedings) issues of the 2006 - 2017 American Economic Review. The fraction of papers with an exemption from the data policy is steadily rising over time, with more than 45 percent of empirical papers exempted from the policy in 2016. 


\section{Data Appendix}

\section{Figure 1}

The data for Figure 1 come from Brodeur et al. (2016), who collect data on every paper in the American Economic Review, Journal of Political Economy, and The Quarterly Journal of Economics that appeared between 2005 and 2011 and included at least one empirical test. The dataset excludes the May American Economic Review Papers and Proceedings (AER PEP) issues. The authors report, among other information related to each empirical test in the sample of papers, the journal, issue, year, major field (microeconomics or macroeconomics), and secondary field (ie, labor or public), and whether the paper was an RCT, a lab experiment, or "other". To construct Figure 1, I collapse these data to the year level, and construct the fraction of papers (overall, by journal, or by microeconomics field, in panels $\mathrm{A}, \mathrm{B}$, and $\mathrm{C}$, respectively) that is categorized as "other."

\section{Figure 2}

To construct Figure 2, I collected data from the American Economic Review Papers and Proceedings from 2006 to 2017, which describe adherence with the journal's data policy in papers published between 2005 and 2016. The American Economic Review first enacted its data policy, requiring researchers to post replication data and code, in 2004. The policy was strengthened in 2005 to include more systematic enforcement and to have "tighter rules for exemptions. Excemptions are generally granted only if the data can be accesssed by other researchers in some alternative fashion" (Moffitt (2006)). Each AER PESP between 2005 and 2017 includes a report 
of the editor, which contains a table with data policy statistics 15 These data report the number of papers, the number of papers with data, the number of exemptions from the data policy, and the number of non-compliant papers in each issue of the journal published in the preceding year (ie, the 2006 AER PEP has data from papers published in 2005). I collapse the data to the annual level, and present the fraction of papers with data that received an exemption from the data policy in each year, in part because the American Economic Review went from publishing quarterly to bimonthly to monthly over this time period.

15. I hand-coded these data, as they were not already available in spreadsheet form from the American Economic Association's website. In particular, I collected data from Moffitt $(2006,2007$, 2008, 2009, 2010, and 2011) and Goldberg (2012, 2013, 2014, 2015, 2016, and 2017). 


\section{Appendix References}

Brodeur, Abel, Mathias Lé, Marc Sangnier, and Yanos Zylberberg. 2016. "Star Wars: The Empirics Strike Back." American Economic Journal: Applied Economics 8 (1): $1-32$.

Goldberg, Pinelopi Koujianou. 2012. "Report of the Editor: American Economic Review." American Economic Review 102 (3): 653-665.

— 2013. "Report of the Editor: American Economic Review." American Economic Review 103 (3): 701-712.

- 2014. "Report of the Editor: American Economic Review." American Economic Review 104 (5): 621-631.

- 2015. "Report of the Editor: American Economic Review." American Economic Review 105 (5): 698-710.

—. 2016. "Report of the Editor: American Economic Review." American Economic Review 106 (5): 700-712.

- 2017. "Report of the Editor: American Economic Review." American Economic Review 107 (5): 699-712.

Moffitt, Robert A. 2006. "Report of the Editor: American Economic Review." American Economic Review 96 (2): 497-509.

. 2007. "Report of the Editor: American Economic Review." American Economic Review 97 (2): 542-554.

— 2008. "Report of the Editor: American Economic Review." American Economic Review 98 (2): 581-590.

- 2009. "Report of the Editor: American Economic Review." American Economic Review 99 (2): 660-670.

- 2010. "Report of the Editor: American Economic Review." American Economic Review 100 (2): 667-676.

. 2011. "Report of the Editor: American Economic Review (with Appendix by Philip J. Glandon)." American Economic Review 101 (3): 684-693. 